https://helda.helsinki.fi

\title{
Hubble law and brane matter after ekpyrosis
}

\section{Enqvist, Kari}

2001-10-05

Enqvist , K , Keski-Vakkuri , E \& Räsänen , S 2001, ' Hubble law and brane matter after ekpyrosis ' , Nuclear Physics, Section B , vol. 614 , no. 1 , pp. 388-401 . https://doi.org/10.1016/S0550-3213(01)004

http://hdl.handle.net/10138/310199

https://doi.org/10.1016/S0550-3213(01)00412-6

cc_by

acceptedVersion

Downloaded from Helda, University of Helsinki institutional repository.

This is an electronic reprint of the original article.

This reprint may differ from the original in pagination and typographic detail.

Please cite the original version. 
hep-th/0106282

HIP-2001-28/TH

June 29, 2001

\title{
Hubble Law and Brane Matter after Ekpyrosis
}

\author{
Kari Enqvist地, Esko Keski-Vakkuri活 and Syksy Räsänen巴 \\ ${ }^{1,2}$ Helsinki Institute of Physics \\ P.O. Box 64, FIN-00014 University of Helsinki, Finland \\ and \\ ${ }^{1,3}$ Department of Physics \\ P.O. Box 64, FIN-00014 University of Helsinki, Finland
}

\begin{abstract}
We study brane matter in the ekpyrotic scenario and observe that in order to obtain standard gravity on the visible brane, the tension of the visible brane should be positive. If the sizes of both the fifth dimension and the Calabi-Yau threefold are fixed, the Israel junction conditions do not allow time-dependent brane matter. Relaxing this constraint, it is possible to obtain approximately standard cosmology on the visible brane, with small corrections due to possible time-dependence of the Calabi-Yau threefold.
\end{abstract}

\footnotetext{
${ }^{1}$ E-mail: kari.enqvist@helsinki.fi

${ }^{2}$ E-mail: esko.keski-vakkuri@hip.fi

${ }^{3}$ E-mail: syksy.rasanen@helsinki.fi
} 


\section{Introduction}

Recently, a novel cosmological scenario based on colliding branes was proposed [囬]. According to [1], this scenario, entitled "the ekpyrotic universe", is based on the HořavaWitten model of M-theory on a $S^{1} / Z_{2}$ orbifold [2], compactified to five dimensions on a Calabi-Yau threefold. The five-dimensional spacetime is bounded by two orbifold planes, one corresponding to our universe (the visible brane), and the other one to a hidden universe. Between the boundary branes, a third brane travels across the bulk spacetime, eventually colliding with and dissolving into the visible brane. The collision, called "ekpyrosis", heats up the visible brane, providing initial conditions for the hot Big Bang. This model was presented as an alternative to the inflationary scenario, resolving the horizon, flatness, and monopole puzzles without involving superluminal expansion. It was even suggested that the model gives a prediction for a strongly blue gravitational wave spectrum, hoped to be detectable in future experiments.

This is an interesting proposal; debate and more detailed examinations have already begun [3, 4, 5, 6, 7]. While many of the key issues, such as the initial configuration of the branes, the dynamics of the bulk brane, and the details of the collision of the bulk brane with the visible brane involve a detailed understanding of the physics of string/M-theory, there are other features which are more tractable and can be studied in the framework of the effective field theory limit of five-dimensional heterotic M-theory 8, 9]. In this paper, we will investigate the cosmology on the visible brane after the collision in the effective field theory context.

One of the debated issues is whether the visible brane should have positive or negative tension [3, 4, 5, 7]. We propose that a simple way to test that is to examine the Hubble law in the post-ekpyrosis era. In the context of the related Randall-Sundrum model [10], the Hubble law has been extensively investigated, and it is known that obtaining (nearly) standard cosmology requires that the visible brane has positive tension [12, 13, 14, 15]. Another feature known from investigations of the Randall-Sundrum model is that the Israel junction conditions do not necessarily allow generic brane matter [16, 17, 18, 19]. These issues are relevant in the ekpyrotic scenario as well - do the junction conditions allow generic time-dependent matter on the visible brane, and can a (nearly) standard Hubble law be recovered?

As we discuss in sections 2.2 and 2.3 , both of the above requirements may be met, but at a price. The junction conditions allow time-dependent matter on the visible brane only if one is willing to allow time evolution for the breathing modulus of the Calabi-Yau threefold or the proper distance of the orbifold planes (or possibly some other moduli).

\footnotetext{
${ }^{1} \mathrm{Or}$ is at least easiest when; see [1] for an alternative, which however is not realised in the ekpyrotic scenario.
} 
An almost standard Hubble law can be recovered if the tension of the visible brane is positive, as in the Randall-Sundrum model. The required cancellations originate from the (near) BPS property of the brane configuration, in the spirit of [20].

The requirement of positive tension for the visible brane does not violate the principles of the ekpyrotic scenario, but helps to narrow the range of possibilities for its realization (see [3, 团 for discussion). The result on brane matter calls for additional analysis of the dynamics of the effective field theory with time-dependent moduli.

\section{Brane cosmology after ekpyrosis}

\section{$2.1 \quad$ The set-up}

The action and the metric. The five-dimensional effective action of heterotic Mtheory is quite complicated [8, 9]. In [四], a simplified action used as an example of the realization of the ekpyrotic scenario was given ast

$$
\begin{gathered}
S_{\text {het }}=\frac{M_{5}^{3}}{2} \int_{\mathcal{M}_{5}} d^{5} x \sqrt{-g}\left(R-\frac{1}{2} \partial_{A} \phi \partial^{A} \phi-\frac{1}{5 !} e^{2 \phi} \mathcal{F}_{A B C D E} \mathcal{F}^{A B C D E}\right) \\
-\sum_{i=1}^{2} 3 \alpha_{i} M_{5}^{3} \int_{\mathcal{M}_{4}^{(i)}} d^{4} \xi_{(i)}\left(\sqrt{-h_{(i)}} e^{-\phi}-\frac{1}{4 !} \epsilon^{\mu \nu \kappa \lambda} \sqrt{\frac{2}{3}} \mathcal{A}_{A B C D} \partial_{\mu} X_{(i)}^{A} \partial_{\nu} X_{(i)}^{B} \partial_{\kappa} X_{(i)}^{C} \partial_{\lambda} X_{(i)}^{D}\right),
\end{gathered}
$$

where $M_{5}$ is the five-dimensional Planck mass, $R$ is the five-dimensional Ricci scalar, $\phi$ is the breathing modulus of the Calabi-Yau threefold and $\mathcal{A}_{A B C D}$ is a four-form gauge field with field strength $\mathcal{F}=d \mathcal{A}$. The Latin indices run from 0 to 4 and the Greek indices run from 0 to 3 . The spacetime is a five-dimensional manifold $\mathcal{M}_{5}=\mathcal{M}_{4} \times S_{1} / Z_{2}$ with coordinates $x^{A}$. The four-dimensional manifolds $\mathcal{M}_{4}^{(i)}, i=1,2$, are the end-of-the-world orbifold planes, called the visible and hidden branes respectively, with internal coordinates $\xi_{(i)}^{\mu}$ and tensions $\alpha_{i} M_{5}^{3}$. The tensor $g_{A B}$ is the metric on $\mathcal{M}_{5}, h_{\mu \nu}^{(i)}$ is the induced metric on $\mathcal{M}_{4}^{(i)}$, and $g$ and $h_{(i)}$ are their determinants. The functions $X_{(i)}^{A}\left(\xi_{(i)}^{\mu}\right)$ are the coordinates in $\mathcal{M}_{5}$ of a point on $\mathcal{M}_{4}^{(i)}$ with coordinates $\xi_{(i)}^{\mu}$. In other words, $X_{(i)}^{A}\left(\xi_{(i)}^{\mu}\right)$ encode the embedding of the branes into $\mathcal{M}_{5}$. The symbol $\epsilon^{\mu \nu \kappa \lambda}$ is the antisymmetrization operator, with $\epsilon^{0123}=1$.

We are interested in post-ekpyrosis cosmology, so the bulk brane present in [1] has already collided with and dissolved into the visible brane. We will not consider ekpyrosis (the collision); following [1] we assume that matter is created on the visible brane and so add to the action the term

$$
S_{\text {matter }}=\int_{\mathcal{M}_{4}^{(1)}} d^{4} \xi_{(1)} \sqrt{-h_{(1)}} \mathcal{L}_{\text {matter }}
$$

\footnotetext{
${ }^{2}$ In [1], there seems to be a factor $\sqrt{2 / 3}$ missing in front of $\mathcal{A}_{A B C D}$. This is explained in [7].
} 
We will neglect the possibility of bending of the branes and take their embedding to be the same as in the BPS state,

$$
X_{(i)}^{A}\left(\xi_{(i)}^{\mu}\right)=\left(x^{0}, x^{1}, x^{2}, x^{3}, y_{i}\right)
$$

with $y_{1}=0, y_{2}=R$. We will consider the following metric $\left(t \equiv x^{0}\right)$ :

$$
d s^{2}=-n(t, y)^{2} d t^{2}+a(t, y)^{2} \sum_{k=1}^{3}\left(d x^{k}\right)^{2}+b(t, y)^{2} d y^{2}
$$

Homogeneity and isotropy with respect to the spatial directions parallel to the branes would allow a $d t d y$-crossterm. However, following the custom in the literature, we will neglect the crossterm. We will denote partial derivatives with respect to $t$ and $y$ by dots and primes. Given the embedding (3), the total action reads

$$
\begin{aligned}
S= & M_{5}^{3} \int_{\mathcal{M}_{5}} d^{5} x \sqrt{-g}\left[\frac{1}{2} R-\frac{1}{4} \partial_{A} \phi \partial^{A} \phi-\frac{1}{2} \frac{1}{5 !} e^{2 \phi} \mathcal{F}_{A B C D E} \mathcal{F}^{A B C D E}\right. \\
& \left.+\sum_{i=1}^{2} \delta\left(y-y_{i}\right) b^{-1}\left(-3 \alpha_{i} e^{-\phi}+\left(-h_{(i)}\right)^{-1 / 2} \sqrt{6} \alpha_{i} \mathcal{A}_{0123}+\delta_{i 1} M_{5}^{-3} \mathcal{L}_{\text {matter }}\right)\right]
\end{aligned}
$$

Because of the $S_{1} / Z_{2}$ orbifold structure of $\mathcal{M}_{5}$, the first $y$-derivatives of the metric and of the fields $\phi$ and $\mathcal{A}_{A B C D}$ are discontinuous at the brane locations, and their second derivatives contain delta functions related to the discontinuities. For example, the delta function part of $a^{\prime \prime}$ is 21

$$
\begin{aligned}
\left.a^{\prime \prime}\right|_{\delta} & =\sum_{i=1,2} \delta\left(y-y_{i}\right)\left[a^{\prime}\right] \\
& =\sum_{i=1,2} \delta\left(y-y_{i}\right)(-1)^{i+1} 2 a_{c}^{\prime},
\end{aligned}
$$

where $\left[a^{\prime}\right]$ is the discontinuity of $a^{\prime},\left[a^{\prime}(y)\right]:=\lim _{\varepsilon \rightarrow 0}\left(a^{\prime}(y+\varepsilon)-a^{\prime}(y-\varepsilon)\right)$, and $a_{c}^{\prime}$ is the continuous part of $a^{\prime}$. An identical relation holds for $n, \phi$, and $\mathcal{A}_{A B C D}$.

The equations of motion. From the action (5) we obtain the following field equations for $\phi$ and $\mathcal{A}_{A B C D}$ :

$$
\begin{aligned}
& \square \phi-\frac{2}{5 !} e^{2 \phi} \mathcal{F}_{A B C D E} \mathcal{F}^{A B C D E}+\sum_{i=1}^{2} \delta\left(y-y_{i}\right) b^{-1} 6 \alpha_{i} e^{-\phi}=0 \\
& D_{M}\left(e^{2 \phi} \mathcal{F}^{M A B C D}\right)+\delta_{0}^{[A} \delta_{1}{ }^{B} \delta_{2}{ }^{C} \delta_{3}{ }^{D]} \sum_{i=1}^{2} \delta\left(y-y_{i}\right)(-g)^{-1 / 2} \sqrt{6} \alpha_{i}=0,
\end{aligned}
$$

\footnotetext{
${ }^{3}$ Note that our $n$ and $a$ are not the same lapse function and expansion factor as the ones denoted by $n$ and $a$ in [1].
} 
where $D_{M}$ is the covariant derivative. Writing the delta function parts of the second $y$-derivative of $\phi$ and the first $y$-derivative of $\mathcal{F}_{M A B C D}$ explicitly, we have

$$
\begin{aligned}
-n^{-2}\left[\ddot{\phi}+\left(-\frac{\dot{n}}{n}+3 \frac{\dot{a}}{a}+\frac{\dot{b}}{b}\right) \dot{\phi}\right] & \\
+b^{-2}\left[\phi_{c}^{\prime \prime}+\left(\frac{n_{c}^{\prime}}{n}+3 \frac{a_{c}^{\prime}}{a}-\frac{b_{c}^{\prime}}{b}\right) \phi_{c}^{\prime}\right]+2 n^{-2} a^{-6} b^{-2} e^{2 \phi}\left(\mathcal{F}_{0123 y c}\right)^{2} & =0 \\
\partial_{t}\left(n^{-1} a^{-3} b^{-1} e^{2 \phi} \mathcal{F}_{0123 y c}\right) & =0 \\
\partial_{y}\left(n^{-1} a^{-3} b^{-1} e^{2 \phi} \mathcal{F}_{0123 y c}\right) & =0 \\
\delta\left(y-y_{i}\right)\left((-1)^{i+1} \phi_{c}^{\prime}+3 b \alpha_{i} e^{-\phi}\right) & =0 \\
\delta\left(y-y_{i}\right)\left(2(-1)^{i} n^{-1} a^{-3} b^{-1} e^{2 \phi} \mathcal{F}_{0123 y c}+\sqrt{6} \alpha_{i}\right) & =0 .
\end{aligned}
$$

In what follows, we suppress the subscript $c$ for convenience; it should be clear from the context whether it is the function or its continuous part that is meant. Solving for the field strength $\mathcal{F}_{0123 y}$, we get

$$
\begin{aligned}
\mathcal{F}_{0123 y} & =-\sqrt{\frac{3}{2}} \alpha n a^{3} b e^{-2 \phi} \\
\alpha_{i} & =(-1)^{i} \alpha \\
-n^{-2}\left[\ddot{\phi}+\left(-\frac{\dot{n}}{n}+3 \frac{\dot{a}}{a}+\frac{\dot{b}}{b}\right) \dot{\phi}\right] & \\
+b^{-2}\left[\phi^{\prime \prime}+\left(\frac{n^{\prime}}{n}+3 \frac{a^{\prime}}{a}-\frac{b^{\prime}}{b}\right) \phi^{\prime}\right]+3 \alpha^{2} e^{-2 \phi} & =0 \\
\delta\left(y-y_{i}\right)\left(\phi^{\prime}-3 b \alpha e^{-\phi}\right) & =0 .
\end{aligned}
$$

We follow the convention of [1]: the hidden brane has tension $\alpha M_{5}^{3}$ and the visible brane has tension $-\alpha M_{5}^{3}$. In [1], $\alpha$ was positive so that the visible brane had negative tension; we leave the sign undetermined for the time being.

The Einstein equation. In addition to the field equations of $\phi$ and $\mathcal{A}_{A B C D}$, we must take into account the Einstein equation

$$
G_{A B}=\frac{1}{M_{5}^{3}} T_{A B} .
$$


In component form, the Einstein equation from the action (5) reads

$$
\begin{aligned}
G_{t}^{t}= & \frac{3}{b^{2}}\left[\frac{a^{\prime \prime}}{a}+\frac{a^{\prime}}{a}\left(\frac{a^{\prime}}{a}-\frac{b^{\prime}}{b}\right)\right]-\frac{3}{n^{2}} \frac{\dot{a}}{a}\left(\frac{\dot{a}}{a}+\frac{\dot{b}}{b}\right) \\
= & -\frac{1}{4} n^{-2} \dot{\phi}^{2}-\frac{1}{4} b^{-2} \phi^{\prime 2}-\frac{3}{4} \alpha^{2} e^{-2 \phi}-\frac{1}{M_{5}^{3}} \sum_{i=1}^{2} \delta\left(y-y_{i}\right) b^{-1} \rho_{b(i)} \\
G_{k}^{k}= & \frac{1}{b^{2}}\left[2 \frac{a^{\prime \prime}}{a}+\frac{n^{\prime \prime}}{n}+\frac{a^{\prime}}{a}\left(\frac{a^{\prime}}{a}+2 \frac{n^{\prime}}{n}\right)-\frac{b^{\prime}}{b}\left(\frac{n^{\prime}}{n}+2 \frac{a^{\prime}}{a}\right)\right] \\
& -\frac{1}{n^{2}}\left[2 \frac{\ddot{a}}{a}+\frac{\ddot{b}}{b}+\frac{\dot{a}}{a}\left(\frac{\dot{a}}{a}-2 \frac{\dot{n}}{n}\right)+\frac{\dot{b}}{b}\left(2 \frac{\dot{a}}{a}-\frac{\dot{n}}{n}\right)\right] \\
= & \frac{1}{4} n^{-2} \dot{\phi}^{2}-\frac{1}{4} b^{-2} \phi^{\prime 2}-\frac{3}{4} \alpha^{2} e^{-2 \phi}+\frac{1}{M_{5}^{3}} \sum_{i=1}^{2} \delta\left(y-y_{i}\right) b^{-1} p_{b(i)} \\
G_{y}^{y}= & \frac{3}{b^{2}} \frac{a^{\prime}}{a}\left(\frac{a^{\prime}}{a}+\frac{n^{\prime}}{n}\right)-\frac{3}{n^{2}}\left[\frac{\ddot{a}}{a}+\frac{\dot{a}}{a}\left(\frac{\dot{a}}{a}-\frac{\dot{n}}{n}\right)\right] \\
= & \frac{1}{4} n^{-2} \dot{\phi}^{2}+\frac{1}{4} b^{-2} \phi^{\prime 2}-\frac{3}{4} \alpha^{2} e^{-2 \phi} \\
G_{t y}= & 3\left(\frac{n^{\prime}}{n} \frac{\dot{a}}{a}+\frac{a^{\prime}}{a} \frac{\dot{b}}{b}-\frac{\dot{a}^{\prime}}{a}\right)=\frac{1}{2} \dot{\phi} \phi^{\prime}
\end{aligned}
$$

where $\rho_{b(i)}$ and $p_{b(i)}$ are the energy density and pressure of brane $i$ :

$$
\begin{aligned}
& \rho_{b(i)}=\delta_{i 1} \rho_{m}+3 M_{5}^{3} \alpha_{i} e^{-\phi} \\
& p_{b(i)}=\delta_{i 1} p_{m}-3 M_{5}^{3} \alpha_{i} e^{-\phi} .
\end{aligned}
$$

The terms $\rho_{m}$ and $p_{m}$ are the contribution of brane matter $\left(\mathcal{L}_{\text {matter }}\right)$. Note that under the assumption of homogeneity and isotropy, the energy-momentum tensor of brane matter necessarily has the ideal fluid form. The delta function part of (15) reads

$$
\begin{aligned}
\left.3 \frac{1}{b} \frac{a^{\prime \prime}}{a}\right|_{\delta} & =-\frac{1}{M_{5}^{3}} \sum_{i=1}^{2} \delta\left(y-y_{i}\right) \rho_{b(i)} \\
\left.\frac{1}{b}\left(2 \frac{a^{\prime \prime}}{a}+\frac{n^{\prime \prime}}{n}\right)\right|_{\delta} & =\frac{1}{M_{5}^{3}} \sum_{i=1}^{2} \delta\left(y-y_{i}\right) p_{b(i)} .
\end{aligned}
$$

Using (6), the above equations can be rewritten as

$$
\begin{aligned}
\left.(-1)^{i+1} \frac{1}{b} \frac{a^{\prime}}{a}\right|_{y=y_{i}} & =-\frac{1}{6 M_{5}^{3}} \rho_{b(i)} \\
\left.(-1)^{i+1} \frac{1}{b} \frac{n^{\prime}}{n}\right|_{y=y_{i}} & =\frac{1}{6 M_{5}^{3}}\left(2 \rho_{b(i)}+3 p_{b(i)}\right) .
\end{aligned}
$$




\subsection{No brane matter in a static configuration}

As the basis of the realization of the ekpyrotic scenario, [1] used the metric of the unique Poincare invariant BPS solution of the simplified action (何),

$$
d s^{2}=D(y)\left(-N^{2} d t^{2}+A^{2} \sum_{k=1}^{3}\left(d x^{k}\right)^{2}\right)+B^{2} D(y)^{4} d y^{2},
$$

where $D(y)=\alpha y+C$ and $N, A, B$ and $C$ are constants. The functions $\phi$ and $\mathcal{F}_{0123 y}$ can be found in [1, [7]. Even allowing for time-dependence in the parameters $N, A, B$ and $C$, we see that $a^{\prime} / a=n^{\prime} / n$. From (18) it then follows that $\rho_{m}+p_{m}=0$, which implies that $\dot{\rho}_{m}=0$.

Even the time-dependent extension of the BPS metric does not allow for time-dependent matter on the brane. Actually, it is a well-known feature of the Randall-Sundrum model that a factorisable metric cannot support time-dependent brane matter [16, 17. More generally, it is known that constraints on brane matter may arise in configurations which are static, meaning that the branes are at rest and the size of the fifth dimension is fixed $(\dot{b}=0)$ [18, 19]. We will now show that this feature is found in the ekpyrotic model as well.

We will look for constraints on brane matter in static configurations. By a "static configuration" we mean a stabilized fifth dimension (in coordinate systems where the branes are at rest, $\dot{b}=0)$ and a stabilized Calabi-Yau threefold $(\dot{\phi}=0)$. The motivation for the second condition is that the breathing modulus $\phi$ is related to the Newton's constant measured on the brane. Therefore, obtaining standard four-dimensional gravity on the brane would seem to require $\dot{\phi}$ to be quite small.p

For a static configuration, the equation of motion of $\phi$, (12), reads

$$
b^{-2}\left(\phi^{\prime \prime}+\left(\frac{n^{\prime}}{n}+3 \frac{a^{\prime}}{a}-\frac{b^{\prime}}{b}\right) \phi^{\prime}\right)+3 \alpha^{2} e^{-2 \phi}=0 .
$$

Since $\dot{b}=0$ and $\dot{\phi}=0$, the time derivative of the above equation gives

$$
\phi^{\prime} \partial_{t}\left(\frac{n^{\prime}}{n}+3 \frac{a^{\prime}}{a}\right)=0 \text {. }
$$

We now consider the location of the visible brane, $y=y_{1}$. The function $\phi^{\prime}$ cannot be zero at the branes, as we see from (13). Relating $n^{\prime} / n$ and $a^{\prime} / a$ to the density and pressure of brane matter, using (18), we obtain the condition

$$
\dot{\rho}_{m}-3 \dot{p}_{m}=0 \text {. }
$$

\footnotetext{
${ }^{4}$ Brane matter satisfies the ordinary four-dimensional conservation law, as we will see in the next section.

${ }^{5}$ The relation between $\phi$ and Newton's constant will be considered in the next section.
} 
Thus, the only matter allowed on the visible (or hidden) brane by the equation of motion of $\phi$ is a combination of radiation and vacuum energy. However, from the Einstein equation one can deduce that even radiation is disallowed. 0 Hence a static configuration cannot support time-dependent brane matter. One may ask if turning on additional fields not present in the simplified action (5) could help the situation. However, additional fields can only help if they couple to the breathing modulus $\phi$ in such a manner as to contribute new time-dependent terms to its equation of motion.

This is our first result: in order to have time-dependent brane matter, one must have $\dot{b} \neq 0$ or $\dot{\phi} \neq 0$. The time-dependence of brane matter is related to the time-dependence of the size of the hidden dimensions. Turning the argument around, time-dependent brane matter will cause either the fifth dimension or the Calabi-Yau threefold to vary in tune with the expansion of the visible universe.

In the limit of vanishing brane matter, we would expect to find a time-independent solution such as the BPS solution of [1]. Then not only the presence but also the amount of time-dependence in $b$ and $\phi$ would seem to depend on brane matter. One may ask whether it is possible to recover approximately standard cosmology on the brane at all, or whether the time-dependence of the extra dimensions will always spoil attempts to obtain the usual Friedmann-Robertson-Walker equations with brane matter as the dominant source. If this is possible, it is then interesting to ask whether the small deviations from standard cosmology will be observable.

\subsection{Cosmology on the brane}

Hubble law on the branes. We will now consider the cosmological evolution as seen by an observer on the visible brane (the results would also apply to the hidden brane, were it to contain matter). In this section, we assume that it is possible to introduce generic time-dependent matter on the visible brane (by a suitable generalization of the scenario, as discussed above), and proceed to examine if standard cosmology can be recovered.

The topic of brane cosmology has been extensively studied in the context of the Randall-Sundrum model [12-25], to mention a few references, and some of the results of these investigations are relevant also to the ekpyrotic model. Cosmology on the branes in the case of a scalar field with a potential in the bulk and on the branes has been studied in [26-29].

Bulk matter content (and more generally, bulk curvature) will in general affect cosmology on the branes. Therefore, in order to obtain the expansion laws on the brane, one would (in general) first have to solve the Einstein equation (or at least the field equations) in the bulk. We will however follow an easier route and analyze only the brane part of

\footnotetext{
${ }^{6}$ To see this, it is most straightforward to consider the combination $G^{t}{ }_{t}+3 G^{k}{ }_{k}-2 G^{y}{ }_{y}$ and use (21).
} 
the equations. This shorter road has two caveats to keep in mind. First, in the brane equations there will appear bulk fields as well as a radiation-like term [15, 16, 24, 25], the magnitude of which cannot in general be fixed without reference to the bulk solution. The second, more subtle, point is that there is no guarantee that a solution of the brane equations is also a solution of the full set of (bulk) equations.

We will use the general metric (4), and make no assumption about the time-dependence of $b$ or $\phi$. We assume the visible brane to lie at a fixed position, and with no loss of generality choose coordinates where the lapse function $n\left(t, y_{1}\right)=1$, corresponding to cosmic time on the visible brane. The $y y$ - and $t y$-components of the Einstein equation (15) are

$$
\begin{aligned}
\frac{1}{b^{2}} \frac{a^{\prime}}{a}\left(\frac{a^{\prime}}{a}+\frac{n^{\prime}}{n}\right)-\frac{1}{n^{2}}\left[\frac{\ddot{a}}{a}+\frac{\dot{a}}{a}\left(\frac{\dot{a}}{a}-\frac{\dot{n}}{n}\right)\right] & =\frac{1}{12} n^{-2} \dot{\phi}^{2}+\frac{1}{12} b^{-2} \phi^{2}-\frac{1}{4} \alpha^{2} e^{-2 \phi} \\
\frac{n^{\prime}}{n} \frac{\dot{a}}{a}+\frac{a^{\prime}}{a} \frac{\dot{b}}{b}-\frac{\dot{a}^{\prime}}{a} & =\frac{1}{6} \dot{\phi} \phi^{\prime} .
\end{aligned}
$$

Taking $y=y_{1}=0$, using (18) and taking into account $n(t, 0)=1$, we have

$$
\begin{aligned}
-\frac{1}{36 M_{5}^{6}} \rho_{b(i)}\left(\rho_{b(1)}+3 p_{b(1)}\right)-\left(\frac{\ddot{a}_{0}}{a_{0}}+\frac{\dot{a}_{0}^{2}}{a_{0}^{2}}\right) & =\frac{1}{12} \dot{\phi}_{0}^{2}+\frac{1}{12} b_{0}^{-2} \phi_{0}{ }^{2}-\frac{1}{4} \alpha^{2} e^{-2 \phi_{0}} \\
\dot{\rho}_{b(1)}+3 \frac{\dot{a}_{0}}{a_{0}}\left(\rho_{b(1)}+p_{b(1)}\right) & =M_{5}^{3} b_{0}^{-1} \dot{\phi}_{0} \phi_{0}^{\prime}
\end{aligned}
$$

where $a_{0}(t) \equiv a(t, 0)$ and so on. Expressing $\phi_{0}^{\prime}$ in terms of $\phi_{0}$ by using the equation of motion of $\phi$ on the branes, (13), we have

$$
\begin{aligned}
\left(\frac{\ddot{a}_{0}}{a_{0}}+\frac{\dot{a}_{0}^{2}}{a_{0}^{2}}\right)+\frac{1}{36 M_{5}^{6}} \rho_{b(1)}\left(\rho_{b(1)}+3 p_{b(1)}\right)+\frac{1}{12} \dot{\phi}_{0}^{2}+\frac{1}{2} \alpha^{2} e^{-2 \phi_{0}} & =0 \\
\dot{\rho}_{b(1)}+3 \frac{\dot{a}_{0}}{a_{0}}\left(\rho_{b(1)}+p_{b(1)}\right)+3 M_{5}^{3} \alpha_{1} e^{-\phi_{0}} \dot{\phi}_{0} & =0 .
\end{aligned}
$$

Multiplying (25) by $2 \dot{a}_{0} / a_{0}$ and (26) by $2 \rho_{b(1)} / 36$ and subtracting, and expressing $\rho_{b(1)}$ and $p_{b(1)}$ in terms of $\rho_{m}$ and $p_{m}$ as given by (16), we get

$$
\begin{aligned}
\partial_{t}\left(\frac{\dot{a}_{0}^{2}}{a_{0}^{2}}-\frac{1}{36 M_{5}^{6}} \rho_{b(1)}^{2}+\frac{1}{4} \alpha^{2} e^{-2 \phi_{0}}\right)+4 \frac{\dot{a}_{0}}{a_{0}}\left(\frac{\dot{a}_{0}^{2}}{a_{0}^{2}}-\frac{1}{36 M_{5}^{6}} \rho_{b(1)}^{2}+\frac{1}{4} \alpha^{2} e^{-2 \phi_{0}}\right) & \\
+\frac{1}{6} \frac{\dot{a}_{0}}{a_{0}} \dot{\phi}_{0}^{2}-\frac{1}{6 M_{5}^{3}} \alpha_{1} e^{-\phi_{0}} \dot{\phi}_{0} \rho_{m} & =0 \\
\dot{\rho}_{m}+3 \frac{\dot{a}_{0}}{a_{0}}\left(\rho_{m}+p_{m}\right) & =0 .
\end{aligned}
$$

Integrating (27), we obtain the Hubble law on the visible brane:

$$
\frac{\dot{a}_{0}^{2}}{a_{0}^{2}}=\frac{1}{36 M_{5}^{6}} \rho_{b(1)}^{2}-\frac{1}{4} \alpha^{2} e^{-2 \phi_{0}}+\frac{\mathcal{C}}{a_{0}^{4}}+f(t),
$$


where $\mathcal{C}$ is a constant 7 and $f$ is a solution of the following equation:

$$
\dot{f}+4 \frac{\dot{a}_{0}}{a_{0}} f=\frac{1}{6 M_{5}^{3}} \alpha_{1} e^{-\phi_{0}} \dot{\phi}_{0} \rho_{m}-\frac{1}{6} \frac{\dot{a}_{0}}{a_{0}} \dot{\phi}_{0}^{2} .
$$

Note that when $\dot{\phi}_{0}=0, f$ reduces to the $\mathcal{C}$-term. The Hubble law (29) is a special case of the Hubble law with a bulk scalar field with a general potential considered in 29.

The Hubble law (29) has a quadratic dependence on the total energy density of the visible brane. So the issue is whether we can recover the standard linear dependence on the energy density of brane matter.

Let us first integrate the equation (30). We write $\rho_{m}=\rho_{r}+\rho_{d}$, to separate the contributions of radiation energy density $\rho_{r}\left(\propto a_{0}^{-4}\right)$ and energy density of other brane matter $\rho_{d}$ (e.g. dust). The result for $f$ is

$$
\begin{aligned}
f= & \frac{1}{6 M_{5}^{3}} a_{0}^{-4} \int d t a_{0}^{4} \alpha_{1} e^{-\phi_{0}} \dot{\phi}_{0} \rho_{m}-\frac{1}{6} a_{0}^{-4} \int d t a_{0}^{3} \dot{a}_{0} \dot{\phi}_{0}^{2} \\
= & -\frac{1}{6 M_{5}^{3}} \alpha_{1}\left(e^{-\phi_{0}}-e^{-\phi_{0}\left(t_{0}\right)}\right) \rho_{r}+\frac{1}{6 M_{5}^{3}} a_{0}^{-4} \int d t a_{0}^{4} \alpha_{1} e^{-\phi_{0}} \dot{\phi}_{0} \rho_{d} \\
& -\frac{1}{6} a_{0}^{-4} \int d t a_{0}^{3} \dot{a}_{0} \dot{\phi}_{0}^{2} .
\end{aligned}
$$

Then, we substitute in (29) the brane energy density $\rho_{b(1)}$ in terms of $\rho_{m}$ as given by (16). Combining (28), (29) and (31), we obtain the ekpyrotic generalization of the Friedmann-Robertson-Walker equations on the visible brane:

$$
\begin{aligned}
\frac{\dot{a}_{0}^{2}}{a_{0}^{2}}= & \frac{1}{6 M_{5}^{3}} \alpha_{1} e^{-\phi_{0}\left(t_{0}\right)} \rho_{r}+\frac{1}{6 M_{5}^{3}} \alpha_{1} e^{-\phi_{0}} \rho_{d}+\frac{1}{36 M_{5}^{6}} \rho_{m}^{2}+\frac{\mathcal{C}}{a_{0}^{4}} \\
& +\frac{1}{6 M_{5}^{3}} a_{0}^{-4} \int d t a_{0}^{4} \alpha_{1} e^{-\phi_{0}} \dot{\phi}_{0} \rho_{d}-\frac{1}{6} a_{0}^{-4} \int d t a_{0}^{3} \dot{a}_{0} \dot{\phi}_{0}^{2} \\
\dot{\rho}_{m}+ & 3 \frac{\dot{a}_{0}}{a_{0}}\left(\rho_{m}+p_{m}\right)=0 .
\end{aligned}
$$

Note that the bulk and brane terms proportional to $\alpha^{2} e^{-2 \phi_{0}}$ have cancelled. So the effective cosmological constant equals zero. We will comment on this after considering how to obtain the rest of standard cosmology.

Towards standard cosmology. The brane equations (32) and (33) are to be compared with the standard four-dimensional FRW equations:

$$
\begin{aligned}
& \frac{\dot{a}^{2}}{a^{2}}=\frac{8 \pi}{3} G_{N} \rho_{m} \\
& \dot{\rho}_{m}+3 \frac{\dot{a}}{a}\left(\rho_{m}+p_{m}\right)=0,
\end{aligned}
$$

\footnotetext{
${ }^{7}$ For the bulk origin and interpretation of this term see [16, 24, 25].
} 
where $G_{N}=1 /\left(8 \pi M_{4}^{2}\right)$ is the four-dimensional Newton's constant.

A comparison of (33) and (35) shows that in the ekpyrotic scenario the brane energymomentum tensor satisfies the standard conservation law. This is because the energy lost (or gained) via time-dependence of the term $e^{-\phi_{0}}$ on the brane is exactly compensated by the (continuous part of the) energy flow $T_{t y}$.

Since space terminates at the branes and $T_{t y}=\frac{1}{2} \dot{\phi} \phi^{\prime}, \dot{\phi}_{0}=0$ seems like a natural boundary condition. However, it is not clear to us whether this boundary condition is absolutely necessary. In what follows, we will keep $\dot{\phi}_{0} \neq 0$ (and see that things simplify considerably if $\dot{\phi}_{0}=0$ ).

A comparison of (32) and (34) shows that on the visible brane, the standard Hubble law and thus standard cosmology are recovered to a good approximation, as long as the conditions

$$
\begin{aligned}
G_{N} & =\frac{\alpha_{1}}{16 \pi M_{5}^{3}} e^{-\phi_{0}} \\
\rho_{m} \frac{M_{4}^{2}}{96 \pi M_{5}^{6}} & \ll 1 \\
\dot{\phi}_{0} & \approx 0, \quad e^{\phi_{0}(t)} \approx e^{\phi_{0}\left(t_{0}\right)}
\end{aligned}
$$

are satisfied.

In addition, we should also have $\mathcal{C} / a^{4} \ll \rho_{m} / M_{4}^{2}$. Let us interpret these conditions. First, in order to obtain standard gravity with a positive Newton's constant, the visible brane must have positive tension, $\alpha_{1}=-\alpha>0$, in contrast to the negative tension used in [1]. This is our second result. A similar result is well known in the context of the Randall-Sundrum model [12, 13, 15] (for a systematic account see [14]); in the context of the Hořava-Witten model this was discussed in [27]. Here, our goals were to i) provide an explicit derivation of the Hubble law in the context of the ekpyrotic scenario, and ii) demonstrate how the issue of obtaining standard cosmology is related to the brane tension assignment.

The second condition is required to ensure that the term quadratic in $\rho_{m}$ is subleading with respect to the term linear in $\rho_{m}$. If $M_{4} \sim M_{5}$, as is natural, this condition is easily satisfied: the quadratic term is vanishingly small and has no observable consequences.

The third condition is required for two reasons: first, to have Newton's constant to approximately constant on the brane (or indeed, to be able to define Newton's constant at all), and second, to have the term involving $\dot{\phi}_{0}^{2}$ be small.

Note the novel feature of (32) that the gravitational coupling of radiation is different from the coupling of other types of matter. The time-dependence of $\phi_{0}$ which changes Newton's constant has no effect on the coupling of radiation. This point is also illustrated by the $y y$-component of the Einstein equation, (25). Expressing $\rho_{b(1)}$ and $p_{b(1)}$ in terms 
of $\rho_{m}$ and $p_{m}$ as given by (16), (25) reads

$$
\frac{\ddot{a}_{0}}{a_{0}}+\frac{\dot{a}_{0}^{2}}{a_{0}^{2}}=\frac{1}{12 M_{5}^{3}} \alpha_{1} e^{-\phi_{0}}\left(\rho_{m}-3 p_{m}\right)-\frac{1}{36 M_{5}^{6}} \rho_{m}\left(\rho_{m}+3 p_{m}\right)-\frac{1}{12} \dot{\phi}_{0}^{2} .
$$

We find again that radiation does not feel the time-dependence of $\phi_{0}$. During the radiation dominated era of cosmology, a time-varying $\phi_{0}$ will only produce deviations from standard cosmology via the additional source proportional to $\dot{\phi}_{0}^{2}$. So, we might safely allow a time-dependent $\phi_{0}$ without significantly affecting for example nucleosynthesis. All that is required is $\dot{\phi}_{0}^{2}<\dot{a}_{0}^{2} / a_{0}^{2}$ by a couple of orders of magnitude. Of course, when the equation of state of brane matter begins to significantly deviate from that of radiation, $\dot{\phi}_{0}$ should be rather small to allow standard cosmology.

A comparison of big bang nucleosynthesis and the Newton's law observed today shows that the gravitational coupling of radiation at the time of nucleosynthesis is close to the gravitational coupling of non-relativistic matter observed today [30]. This means that either the coupling of radiation and the coupling of other matter have been almost the same since nucleosynthesis (requiring $\dot{\phi}_{0}=0$ to a good accuracy; see 31] for experimental limits on $\dot{\phi}_{0}$ ) or the gravitional coupling of radiation is an attractor for the general coupling. The first possibility seems more likely.

By performing a partial integration and using (33), we can write (32) as

$$
\begin{aligned}
\frac{\dot{a}_{0}^{2}}{a_{0}^{2}}= & \frac{1}{6 M_{5}^{3}} \alpha_{1} e^{-\phi_{0}\left(t_{0}\right)} \rho_{r}+\frac{1}{36 M_{5}^{6}} \rho_{m}^{2}+\frac{\mathcal{C}}{a_{0}^{4}} \\
& +a_{0}^{-4} \int d t a_{0}^{3} \dot{a}_{0}\left(\frac{1}{6 M_{5}^{3}} \alpha_{1} e^{-\phi_{0}}\left(\rho_{d}-3 p_{d}\right)-\frac{1}{6} \dot{\phi}_{0}^{2}\right) .
\end{aligned}
$$

As the universe expands, the contributions from the brane energy density and pressure (as well as the $\mathcal{C}$-term) approach zero, assuming that $\phi_{0}$ does not decrease so rapidly that $e^{-\phi_{0}} \rho_{m}$ grows. Since the l.h.s. of (40) is positive definite and the term involving $\dot{\phi}_{0}^{2}$ is negative definite, we see that $\dot{\phi}_{0}$ approaches zero as the universe becomes asymptotically empty. This is in agreement with our observation in section 2.2 that the magnitude of $\dot{\phi}$ (if different from zero) is related to the magnitude of $\rho_{m}$.

Comments. As in the well known case of the Randall-Sundrum model, the cosmology on the branes of the ekpyrotic model differs from FRW cosmology in two ways: the square of the Hubble parameter is proportional to the square of the brane energy density and there is a contribution due to bulk curvature. The usual linear dependence is obtained by having a positive cosmological constant on the brane, at the price of having a term involving the cosmological constant squared. $\mathrm{ft}$ is notable that in the ekpyrotic model, this term is

\footnotetext{
${ }^{8}$ For an alternative mechanism, see [11].
} 
exactly cancelled by a contribution from the bulk curvature, leaving a vanishing effective cosmological constant on the brane. See also [27].

This story is familiar from the Randall-Sundrum model. For cosmological solutions with a cosmological constant as the only bulk source, only one particular relation between the bulk and brane cosmological constants gives a vanishing effective cosmological constant on the brane. This apparent fine-tuning has its origin in the BPS conditions [20]. This is also the case in the ekpyrotic model: the relation between the brane tension and charge that characterises BPS solutions also cancels the effective cosmological constant on the brane for cosmological solutions. A different prefactor of $\mathcal{A}_{A B C D}$ in (1) would yield a non-zero effective cosmological constant on the brane.

The behaviour of terms involving bulk curvature that do not cancel against the brane tension, namely the $\mathcal{C}$-term and $\dot{\phi}_{0}$-terms, as well as the value of the gravitational coupling of radiation, can only be provided by the bulk equations. If one imposes the seemingly natural boundary condition $\dot{\phi}_{0}=0$, then the only ambiguity is related to the $\mathcal{C}$-term and (equivalently) to the gravitational coupling of radiation. Then the ekpyrotic brane cosmology looks, in the homogeneous and isotropic approximation, the same as brane cosmology in the Randall-Sundrum model with a cosmological constant as the only bulk source. However, it is not clear whether the condition $\dot{\phi}_{0}=0$ can be imposed: we have observed that it is possible to obtain standard cosmology if $\dot{\phi}_{0}$ is small, but not shown that it actually is small. In section 2.1 we saw that in order to have time-dependent brane matter we must have either $\dot{b} \neq 0$ or $\dot{\phi} \neq 0$, or both. Note that, as in the RandallSundrum model, the time-dependence of $b$ does not directly enter into the brane expansion equations, so that from the point of view of brane cosmology there is no reason to exclude even a radical time-dependence in $b$ (as opposed to $\phi$ ). For these reasons it would be interesting to find an explicit bulk solution for the post-ekpyrosis universe, possibly as a perturbation around the BPS solution.

\section{Discussion}

We have two main results. We have observed that the visible brane must have positive tension in order to support approximately standard gravity and shown that time-dependent brane matter will cause the size of the hidden dimensions to change in time.

In the original example of a realization of the ekpyrotic scenario [1] (version 1) it was implied that the visible brane should, in that setting, have negative tension. In [3] it was argued that the visible brane cannot have negative tension and a variant with the signs of the brane tensions flipped was investigated. The authors of [1] responded that the visible brane could in principle have either positive or negative tension [4], and that in particular the original setting allows for any sign for the tension of the visible brane [1] (version 
2). Explicit constructions with negative tension visible branes were also presented [5]. The debate continued in [7], where it was argued that obtaining standard Hořava-Witten phenomenology requires the visible brane to have positive tension.

Now, it is again argued that the visible brane should have positive tension. However, we emphasize that our argument follows a different path than the previous investigations. We do not study which tension assignments are possible in M-theory or what is the resulting heterotic phenomenology. Rather, we consider the viewpoint of observers on the visible brane and study the gravity seen by them.

It is worth mentioning that the four-dimensional Newton's constant given by the induced Einstein equation on the brane, (36), differs from the one obtained in the usual dimensional reduction [32]: the inverse of the size of the fifth dimension $\pi R$ is replaced by the brane tension $\alpha_{1}$.

It is perhaps fortunate that the size of the fifth dimension does not appear in the Newton's constant measured on the brane. We have seen that time-dependent brane matter will induce time-dependence in the size of the fifth dimension or in the size of the Calabi-Yau threefold. Of course, we have studied only the simplified action used in [1]. The five-dimensional action of heterotic M-theory is much more complicated and can include a plethora of other moduli [8, 9]. It remains to be seen whether this effect is a general feature of the ekpyrotic scenario, present in more complicated settings as well.

By deriving the Hubble law on the visible brane, we have shown that it is possible to obtain approximately standard cosmology (provided that the brane has positive tension). In contrast to the size of the fifth dimension, the size of the Calabi-Yau threefold appears directly in the Hubble law. Its time-dependence may have observable cosmological effects. In particular, Newton's constant may be time-dependent, though curiously the gravitational coupling of radiation is always time-independent. In order to estimate the magnitude of the effects arising from the time-dependence of the Calabi-Yau threefold, it would be interesting to find an explicit solution where one could see the relation between brane matter and the size of the hidden dimensions.

\section{Acknowledgements}

K.E. has been supported in part by the Academy of Finland under the contract 35224 . We thank Vijay Balasubramanian for discussions. E.K-V. would like to thank the University

of Pennsylvania for hospitality at the inception of this work. We also thank Anne Davis and Andrei Linde for comments.

\section{References}


[1] Justin Khoury, Burt A. Ovrut, Paul J. Steinhardt and Neil Turok. The Ekpyrotic Universe: Colliding Branes and the Origin of the Hot Big Bang. hep-th/0103239.

[2] Petr Hořava and Edward Witten. Heterotic and type I string dynamics from elevendimensions. Nucl. Phys. B460: 506, 1996. hep-th/9510209. Eleven-dimensional supergravity on a manifold with boundary. Nucl. Phys. B475: 94, 1996. hepth/9603142.

[3] Renata Kallosh, Lev Kofman and Andrei Linde. Pyrotechnic Universe. hepth/0104073.

[4] Justin Khoury, Burt A. Ovrut, Paul J. Steinhardt and Neil Turok. A Brief Comment on "The Pyrotechnic Universe". hep-th/0105212.

[5] Ron Y. Donagi, Justin Khoury, Burt A. Ovrut, Paul J. Steinhardt and Neil Turok. Visible Branes with Negative Tension in Heterotic M-Theory. hep-th/0105199.

[6] David H. Lyth. The primordial curvature perturbation in the ekpyrotic Universe. hep-ph/0106153.

[7] Renata Kallosh, Lev Kofman, Andrei Linde, and Arkady Tseytlin. BPS branes in cosmology. hep-th/0106241.

[8] Andre Lukas, Burt A. Ovrut, K.S. Stelle and Daniel Waldram. The Universe as a Domain Wall. Phys. Rev. D59: 086001, 1999. hep-th/9803235.

[9] Andre Lukas, Burt A. Ovrut, K.S. Stelle and Daniel Waldram. Heterotic M-theory in Five Dimensions. Nucl. Phys. B552: 246, 1999. hep-th/9806051.

[10] Lisa Randall and Raman Sundrum. A large mass hierarchy from a small extra dimension. Phys. Rev. Lett. 83: 3370, 1999. hep-ph/9905221. An alternative to compactification. Phys. Rev. Lett. 83: 4690, 1999. hep-th/9906064.

[11] Panagiota Kanti, Ian. I. Kogan, Keith A. Olive and Maxim Pospelov. Cosmological 3-brane solutions. newblock Phys. Lett. B468: 31, 1999. hep-ph/9909481. Single-brane cosmological solutions with a stable compact extra dimension. Phys. Rev. D61: 106004, 2000. hep-ph/9912266. Panagiota Kanti, Keith A. Olive and Maxim Pospelov. Static solutions for brane models with a bulk scalar field. Phys. Lett. B481: 386, 2000. hep-ph/0002229. Solving the hierarchy problem in twobrane cosmological models. hep-ph/0005146. Phys. Rev. D62: 126004, 2000. 
[12] Csaba Csáki, Michael Graesser, Christopher Kolda and John Terning. Cosmology of One Extra Dimension with Localized Gravity. Phys. Lett. B462: 34, 1999. hep$\mathrm{ph} / 9906513$.

[13] James M. Cline, Christophe Grojean and Geraldine Servant. Cosmological Expansion in the Presence of an Extra Dimension. Phys. Rev. Lett. 83: 4245, 1999. hep$\mathrm{ph} / 9906523$.

[14] T. Shiromizu, K. Maeda and M. Sasaki. The Einstein equations on the 3-brane world. Phys. Rev. D62: 024012, 2000. gr-qc/9910076.

[15] Pierre Binétruy, Cédric Deffayet, Ulrich Ellwanger and David Langlois. Brane cosmological evolution in a bulk with cosmological constant. Phys. Lett. B477: 285, 2000. hep-th/9910219.

[16] R.N. Mohapatra, A. Pérez-Lorenzana and C.S. de S. Pires. Cosmology of brane-bulk models in five dimensions. Int. J. Mod. Phys. A16: 1431, 2001. hep-ph/0003328.

[17] Julien Lesgourges, Sergio Pastor, Marco Peloso and Lorenzo Sorbo. Cosmology of the Randall-Sundrum model after dilaton stabilization. Phys. Lett. B489: 411, 2000. hep-ph/0004086.

[18] Benjamin Grinstein, Detlef R. Nolte and Witold Skiba. Adding matter to Poincare invariant branes. Phys. Rev. D62: 086006, 2000. hep-th/0005001.

[19] Kari Enqvist, Esko Keski-Vakkuri and Syksy Räsänen. Constraints on brane and bulk ideal fluid in Randall-Sundrum cosmologies. Phys. Rev. D64: 0240XX, 2001. hep-th/0007254.

[20] Klaus Behrndt and Mirjam Cvetič. Supersymmetric Domain-Wall World from D=5 Simple Gauged Supergravity. Phys. Lett. B475: 253, 2000. hep-th/9909058. Francisco A. Brito, Mirjam Cvetič and SangChul Yoon. From a Thick to a Thin Supergravity Domain Wall. Phys. Rev. D (to be published). hep-ph/0105010.

[21] Pierre Binétruy, Cédric Deffayet and David Langlois. Non-conventional cosmology from a brane-universe. Nucl. Phys. B565: 269, 2000. hep-ph/9905012.

[22] Nemanja Kaloper. Bent domain walls as braneworlds. Phys. Rev. D60: 123506, 1999. hep-th/9905210.

[23] Csaba Csáki, Michael Graesser, Lisa Randall and John Terning. Cosmology of brane models with radion stabilization. Phys. Lett. B462: 34, 1999. hep-ph/9911406. 
[24] D. Ida. Brane-world cosmology. JHEP 09: 014, 2000. gr-qc/9912002.

[25] S. Mukohyama, T. Shiromizu and K. Maeda. Global structure of exact cosmological solutions in the brane world. Phys. Rev. D62: 024028, 2000. hep-th/9912287.

[26] Kei-ichi Maeda and David Wands. Dilaton-gravity on the brane. Phys. Rev. D62: 124009, 2000. hep-th/0008188.

[27] A. Mennim and R.A. Battye. Cosmological expansion on a dilatonic brane-world. Class. Quant. Grav. 18: 2171, 2001. hep-th/0008192.

[28] C. van de Bruck, M. Dorca, C.J.A.P. Martins and M. Parry. Cosmological consequences of the brane/bulk interaction. Phys. Lett. B495: 183, 2000. hepth/0009056.

[29] Ph. Brax and A. C. Davis. Cosmological Evolution on Self-Tuned Branes and the Cosmological Constant. JHEP 0105: 007, 2001. hep-th/0104023.

[30] Frank S. Accetta, Lawrence M. Krauss and Paul Romanelli. New limits on the variability of $\mathrm{G}$ from big bang nucleosynthesis. Phys. Lett. B248: 146, 1990.

[31] D. B. Guenther, L.M. Krauss and P. Demarque. Testing the constancy of the gravitational constant using helioseismology. Astrophys. J. 498: 871, 1998.

[32] Edward Witten. Strong Coupling Expansion Of Calabi-Yau Compactification. Nucl. Phys. B471: 135, 1996. hep-th/9602070. 